\title{
Regulation of nucleosome landscape and transcription factor targeting at tissue-specific enhancers by BRGl
}

\author{
Gangqing Hu, ${ }^{1,4}$ Dustin E. Schones, ${ }^{1,4,5}$ Kairong Cui, ${ }^{1,4}$ River Ybarra, ${ }^{2}$ Daniel Northrup, ${ }^{1}$ \\ Qingsong Tang, ${ }^{1}$ Luca Gattinoni, ${ }^{3}$ Nicholas P. Restifo, ${ }^{3}$ Suming Huang, ${ }^{2}$ and Keji Zhao ${ }^{1,6}$ \\ ${ }^{1}$ Laboratory of Molecular Immunology, National Heart, Lung and Blood Institute, National Institutes of Health, Bethesda, Maryland \\ 20892, USA; ${ }^{2}$ Department of Biochemistry and Molecular Biology, University of Florida College of Medicine, Gainesville, Florida 32611 , \\ USA; ${ }^{3}$ Center for Cancer Research, National Cancer Institute, National Institutes of Health, Bethesda, Maryland 20892, USA
}

\begin{abstract}
Enhancers of transcription activate transcription via binding of sequence-specific transcription factors to their target sites in chromatin. In this report, we identify GATAl-bound distal sites genome-wide and find a global reorganization of the nucleosomes at these potential enhancers during differentiation of hematopoietic stem cells (HSCs) to erythrocytes. We show that the catalytic subunit BRGl of BAF complexes localizes to these distal sites during differentiation and generates a longer nucleosome linker region surrounding the GATAl sites by shifting the flanking nucleosomes away. Intriguingly, we find that the nucleosome shifting specifically facilitates binding of TAL1 but not GATAl and is linked to subsequent transcriptional regulation of target genes.
\end{abstract}

[Supplemental material is available for this article.]

Recent studies have suggested that the activation of transcription is correlated with reorganization of nucleosomes at distal enhancers (Schones et al. 2008; He et al. 2010). While it is established that nucleosomes present a major barrier for the access of transcription factors (TFs) to their target sites in chromatin, it remains unclear how the positions of enhancer nucleosomes are regulated to allow for binding of lineage-specific transcription factors during erythroid differentiation.

The mammalian ATP-dependent chromatin-remodeling BAF complexes (Wang et al. 1996) are capable of altering histone-DNA interactions and sliding nucleosomes in vitro (Imbalzano et al. 1994; Gutierrez et al. 2007), as well as regulating the expression of numerous genes in vivo (Liu et al. 2001; Fan et al. 2005; Ho et al. 2009). BRG1 is involved in numerous cellular differentiation programs including T cell, erythrocyte, and neuronal lineages (Zhao et al. 1998; Bultman et al. 2000; Chi et al. 2002; Im et al. 2005; Wu et al. 2007; Jani et al. 2008; Wurster and Pazin 2008; Kim et al. 2009b). It furthermore regulates gene expression programs in response to interferon stimulation (Liu et al. 2002; Cui et al. 2004) and Toll-like receptor signaling pathways (Lai et al. 2009; Ramirez-Carrozzi et al. 2009). In addition to targeting promoter regions, it has been demonstrated that BRG1 is recruited to distal regulatory elements of the CIITA (Ni et al. 2008) and HBB (O'Neill et al. 1999; Im et al. 2005) to mediate their activation by facilitating long-distance chromatin interaction (Kim et al. 2009b). Although it is not clear how BRG1 recognizes specific promoters and enhancers in vivo, it has been demonstrated that the zinc-finger transcription factor GATA1 collaborates with BRG1 to disrupt chromatin (Armstrong et al. 1998; Kadam and Emerson 2003). Recent studies have demonstrated that BRG1 functionally interacts with GATA1 and EKLF in vivo and is critical for DNase I sensitivity, H3 acetylation, and H3K4me2 levels

\footnotetext{
${ }^{4}$ These authors contributed equally to this work. 5 Present address: Department of Cancer Biology, Beckman Research Institute of the City of Hope, Duarte, California 91010, USA. ${ }^{6}$ Corresponding author.

E-mail zhaok@nhlbi.nih.gov.

Article published online before print. Article, supplemental material, and publication date are at http://www.genome.org/cgi/doi/10.1101/gr.121145.111.
}

at the far-upstream major-regulatory element and the $\alpha 2$ promoter of the HBA locus (Kim et al. 2009a).

ATP-dependent chromatin remodeling complexes could slide, restructure, or evict nucleosomes in vitro (Cairns 2007). However, it has not been fully elucidated how the ubiquitously expressed BRG1 regulates the nucleosome organization surrounding the binding sites of tissue-specific transcription factors to allow for their binding during hematopoiesis because direct measurement of nucleosome occupancy and transcription factor binding relative to BRG1 binding in vivo has not been done. In this study, we investigated the function of BRG1 in nucleosome organization at enhancer elements and subsequent transcription factor binding during differentiation of HSCs to the erythrocyte lineage. Our data indicate that BRG1 functions to shift nucleosomes away from the binding sites of GATA1, which facilitates the binding of TAL1 and subsequent transcriptional activation.

\section{Results}

Identification of GATAl-bound enhancers during differentiation of HSCs to erythrocytes

GATA1 is known to bind to erythroid-specific enhancers and act as a transcriptional activator (Cantor and Orkin 2002). To investigate whether and how nucleosome organization changes during the establishment of functional enhancers, we mapped the genomewide binding sites of GATA1 in CD36 ${ }^{+}$erythrocyte precursor cells that were generated by in vitro differentiation of human bone marrow-derived CD133 ${ }^{+}$cells (HSCs) as described previously (Cui et al. 2009). The expression of GATA1 was not detectible in HSCs but highly induced in the $\mathrm{CD}^{2} 6^{+}$cells (Cui et al. 2009). From the ChIP-seq data, we identified a total of 30,126 GATA1 binding sites, the majority $(81.3 \%)$ of which are located away from the immediate promoter region and thus are called "distal" sites in the following analysis. Sequence analysis of the GATA1 binding sites revealed that most of them contained the consensus GATA motif, GATAA. As expected, GATA1 was highly enriched at known enhancers in the locus control region (LCR) of the beta-globin domain and colocalized with H3K4me1 in CD36 ${ }^{+}$cells (Supplemental Fig. S1). 
Interestingly, the number of GATA1 binding sites per gene was positively correlated with gene activation and inversely correlated with gene repression during HSC differentiation (Supplemental Fig. S1). These data confirmed previous reports on GATA1 binding at the enhancers of specific genes and on global scales in erythroid cells (Cheng et al. 2009; Fujiwara et al. 2009; Kim et al. 2009a,b; Yu et al. 2009) and further demonstrated a global correlation of gene activation with GATA1 binding during differentiation of HSCs. Thus, these GATA1-bound genomic regions are potential enhancers of transcription for erythroid differentiation.

\section{Nucleosomes at GATAl enhancers} are repositioned during differentiation of HSCs

To examine the nucleosome organization at enhancers, we mapped the genomewide nucleosome occupancy in both HSCs and $\mathrm{CD}^{2} 6^{+}$cells using MNase-seq as described previously (Schones et al. 2008). As shown in Figure 1A, nucleosomes were well positioned surrounding the binding sites of the constitutively expressed insulator protein, CTCF, and little difference was detected after differentiation. In striking contrast to the CTCF sites, nucleosome levels were higher over the GATA1 sites than the neighboring regions in both the HSCs and CD36 $6^{+}$cells (Fig. 1B). This observation is consistent with a previous study which demonstrated that regulatory elements in the human genome have high affinity for nucleosomes (Tillo et al. 2010). Further inspection revealed that the nucleosomes surrounding the GATA1 binding sites were better positioned in $\mathrm{CD}^{+} 6^{+}$cells (Fig. 1B, blue line) than in HSCs (Fig. 1B, red line), suggesting that a substantial nucleosome reorganization occurs during the differentiation process. To address whether these nucleosome profiles represent average signals of different nucleosome patterns, we further resolved the nucleosome profiles by grouping the GATA1 sites according to their relative location to nucleosomes in HSCs: one group (17.2\%) was directly overlapped with a nucleosome; and the other group $(82.8 \%)$ was located in the linker region. Further analysis of nucleosome profiles surrounding the GATA1 sites in the linker regions revealed a substantial increase of $40 \mathrm{bp}$ in nucleosome repeat length (from 240 to $280 \mathrm{bp})$ surrounding the GATA1 sites during the differentiation (Fig. 1C), indicating that the flanking nucleosomes are shifted away from the GATA1 sites upon its binding. One specific example
A

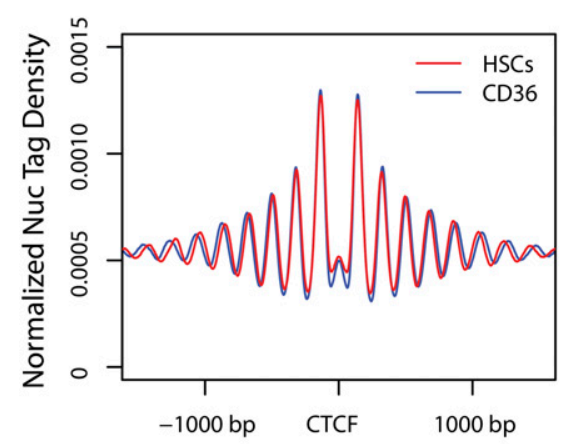

C

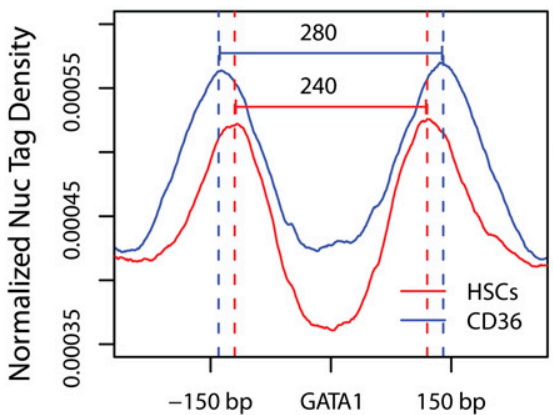

$\mathrm{E}$

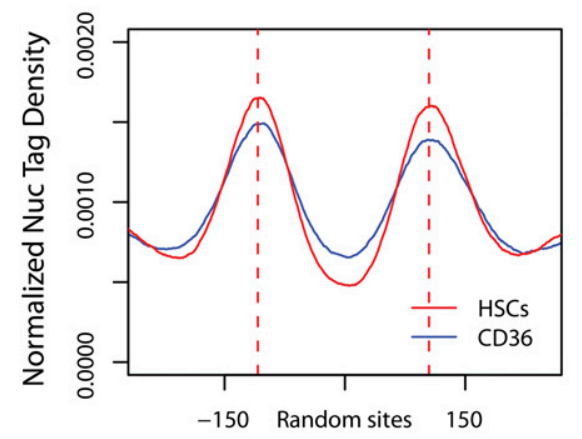

B

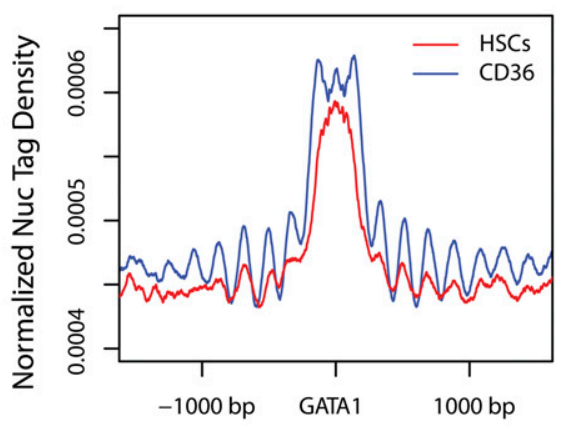

D

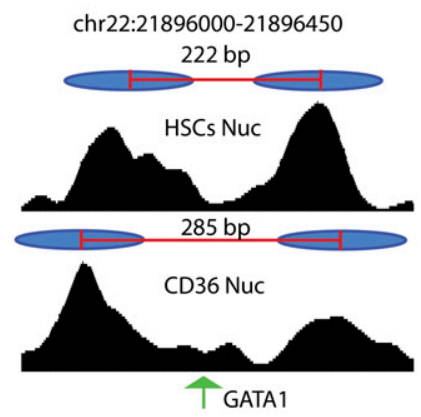

$\mathrm{F}$

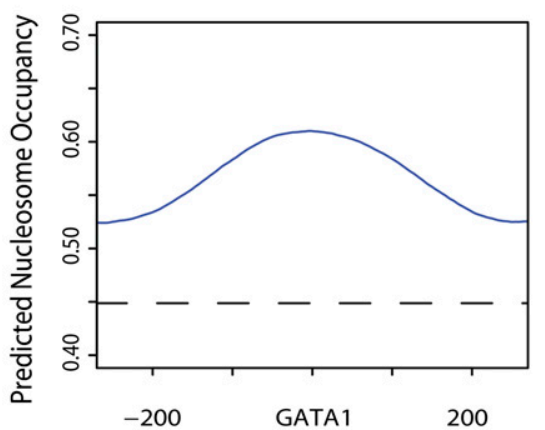

Figure 1. Nucleosomes are reorganized at distal GATA1 binding sites during differentiation of HSCs. (A) Nucleosomes are well positioned surrounding the 18,777 CTCF binding sites shared by both HSCs and $\mathrm{CD} 36^{+}$cells. The nucleosome distribution in $\mathrm{HSCs}$ and $\mathrm{CD} 36^{+}$cells was obtained using MNase-seq, and the normalized nucleosome tag densities were plotted for a 3-kb region surrounding the CTCF binding sites. The genomic averages are about 0.0003 for both libraries. $(B)$ The normalized nucleosome tag densities were plotted for a region of \pm 1500 bp surrounding the 24,491 distal GATA1 sites in HSCs and CD36 ${ }^{+}$cells. (C) The same as in $B$, except that only the 20,284 GATA1 binding sites that are located in the linker region in HSCs are shown for a region of \pm 250 bp surrounding the GATA1 sites. $(D)$ UCSC Genome Browser images showing the nucleosome tag densities in HSCs and CD $36^{+}$cells surrounding a GATA1 binding site on chr22. The inferred nucleosome positions are indicated by filled ovals. The summit of GATA1 binding in $\mathrm{CD}_{3} 6^{+}$cells is indicated by an arrow. $(E)$ The same as in $C$, except for a set of randomly chosen genomic sites located in linker regions. $(F)$ Predicted nucleosome occupancy surrounding the 20,284 distal GATA1 sites that are located in the linker regions in HSCs, based on intrinsic DNA sequence preferences of nucleosome formation (Kaplan et al. 2009). Genomic averages are shown by dashed line. for a GATA1 binding site on chromosome 22 was shown (Fig. 1D). The percentage of linker-associated GATA1 sites could be overestimated since $91 \%$ of randomly selected genomic sites were located in linker regions, which may be caused by insufficient sequencing depth and choice of stringent parameters used to call 
nucleosomes. Nevertheless, with the current data sets, we found that the expansion of linker region is absent at these randomly chosen genomic sites (Fig. 1E). These results indicate that GATA1 binding induces nucleosome reorganization during differentiation of HSCs, similar to the nucleosome reorganization induced by androgen receptor binding in the prostate cancer cell (He et al. 2010). To understand the underlying principle that determines the initial nucleosome organization surrounding GATA1 binding sites in HSCs, we analyzed the nucleosome occupancy estimated based on intrinsic DNA sequence preferences of nucleosome formation (Kaplan et al. 2009). The analysis revealed a higher level of intrinsic nucleosome affinity at the GATA1 sites than the genomic average (Fig. 1F). The reorganization of the nucleosomes surrounding the GATA1 sites during HSC differentiation indicates that trans acting factors are involved in modulating the nucleosome positioning and organization in these functional regulatory genomic regions.

\section{BRGl becomes associated with the GATAl enhancers during differentiation of HSCs}

The BAF complexes alter nucleosome structure and facilitate binding of transcription factors to their target sites in vitro (Imbalzano et al. 1994). To investigate whether they are involved in the observed nucleosome shifting at the GATA1-bound enhancers upon induced differentiation of HSCs, we determined the genome-wide binding sites of BRG1 in both HSCs and CD36 ${ }^{+}$cells. The data indicated that BRG1 was colocalized with GATA1 in the beta-globin domain (Fig. 2A), and significantly increased binding of BRG1 was detected at the GATA1 sites after differentiation (Fig. 2B). In HSCs, only $22 \%$ of the GATA1 sites were associated with BRG1, whereas over $53 \%$ of them were bound by BRG1 in CD36 $6^{+}$cells (Fig. 2C). Interestingly, although the level of BRG1 binding positively correlated with that of both GATA1 and CTCF binding (Fig. 2D), the fold-changes in BRG1 binding during the differentiation were only positively correlated with the binding level of GATA1 but not CTCF (Fig. 2E), which is consistent with GATA1 being involved in recruiting BRG1 to these regions during erythroid differentiation. Interestingly, the positive correlation between GATA1 binding and BRG1 binding is not related to the nucleosome organization prior to differentiation (Supplemental Fig. S2).

\section{BRG1 mediates the nucleosome repositioning at GATAl enhancers}

To test whether BRG1 binding is correlated with nucleosome reorganization at enhancers, we grouped GATA1 sites into four quartiles (I, II, III, and IV) according to the levels of BRG1 binding in $\mathrm{CD}^{+} 6^{+}$cells (IV has the highest BRG1 binding and I has the lowest.) and plotted their nucleosome profiles in HSCs and CD36 $6^{+}$ cells, respectively. While there was not much difference in the nucleosome profiles among these four groups in HSCs, and they all had very similar nucleosome linker lengths (Fig. 3A), a substantially longer distance between the two nucleosomes flanking the GATA1 site (330 bp) was detected for the group (quarter IV) with the highest BRG1 binding as compared to the two bottom groups (quarters I and II, $260 \mathrm{bp}$ ) in $\mathrm{CD}^{+} 6^{+}$cells (Fig. 3B). As a control, we found that the nucleosome shifting was independent of GATA1 binding at the sites that were not bound by BRG1 (Supplemental Fig. S3). To quantify these results for individual loci, we considered GATA1 binding sites that occurred in linkers and had nucleosomes within \pm 200 bps. We then compared the position of the nearest nucleosome relative to the GATA1 site in HSC
A

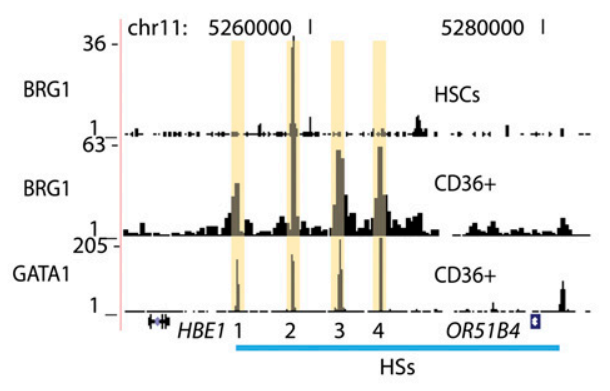

B

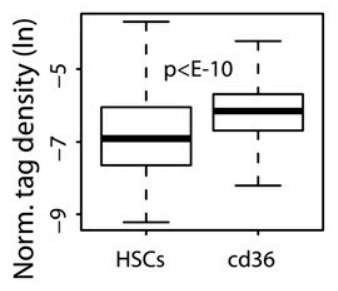

D

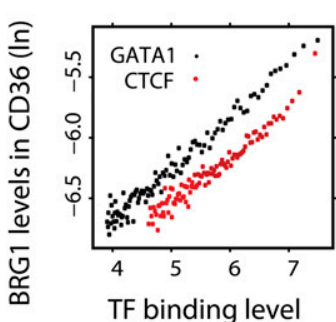

C

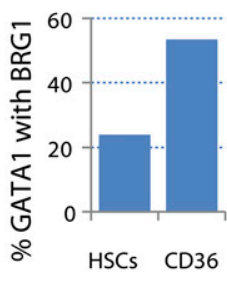

E

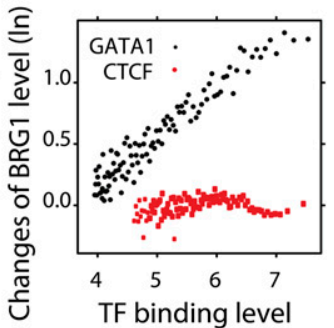

Figure 2. GATA1 binding is positively correlated with BRG1 binding during differentiation of HSCS. (A) UCSC Genome Browser images showing the colocalization of BRG1 with GATA1 in CD36 ${ }^{+}$cells. The four DNase I hypersensitive sites (HS) bound by GATA1 in the LCR of the beta-globin domain are indicated at the bottom. (B) Box-plots of BRG1 density at the 24,491 distal GATA1-bound region in HSCs and CD36 ${ }^{+}$cells. (C) Percentage of GATA1 binding sites that are also bound by BRG1 in HSCs and CD $36^{+}$ cells. $(D)$ Correlation between GATA1 (black dots) and CTCF (red dots) binding levels with that of BRG1 binding in CD $36^{+}$cells. The 30,126 GATA1 or 40,392 CTCF binding sites are binned into groups with equal size of 200 based on the TF binding levels. The densities of BRG1 sequence tags (In) and the TF tag enrichment scores (In) from MACS are then averaged for each group. (E) Correlation between the changes in BRG1 binding from HSCs to CD $36^{+}$cells at GATA1 - or CTCF-bound regions with the levels of GATA1 or CTCF binding in $\mathrm{CD}^{+} 6^{+}$cells. The TF binding sites are binned as in $D$.

and $\mathrm{CD}_{3} 6^{+}$cells. A significantly higher fraction of these sites had the nucleosomes shifted $>10$ bp away from the GATA1 binding sites in the top two groups (quarter III and IV) than the bottom two groups (quarter I and II) (Fig. 3C). These results indicated that the nucleosome shifting at enhancers is positively correlated with BRG1 binding. To test directly whether BRG1 is mediating the nucleosome shifting, we knocked down BRG1 in $\mathrm{CD} 36^{+}$cells using shRNAs (Fig. 3D) and compared the nucleosome profiles in the knockdown and control cells (Fig. 3E). We found that knocking down BRG1 resulted in a reverse shift of nucleosomes that shortened the nucleosome linker region surrounding the GATA1 sites, and the degree of the nucleosome shift was positively correlated with the BRG1 binding level (Fig. 3E). Moreover, the fraction of 
A

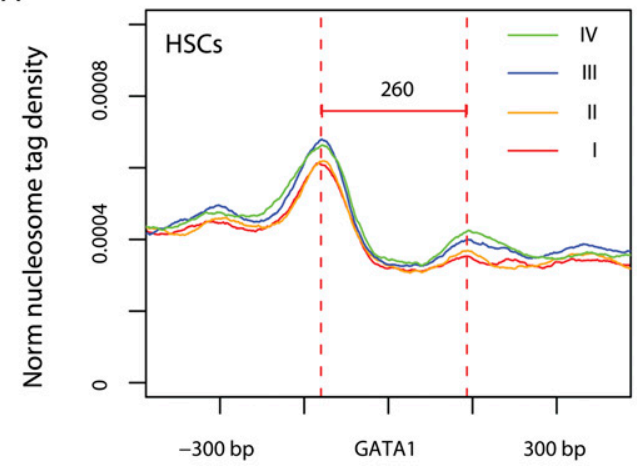

B

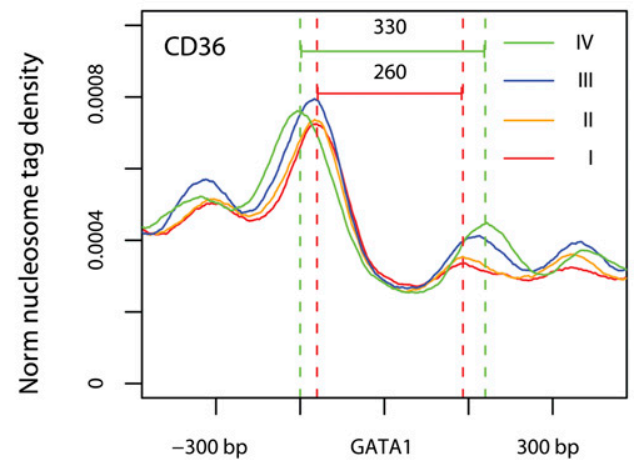

C

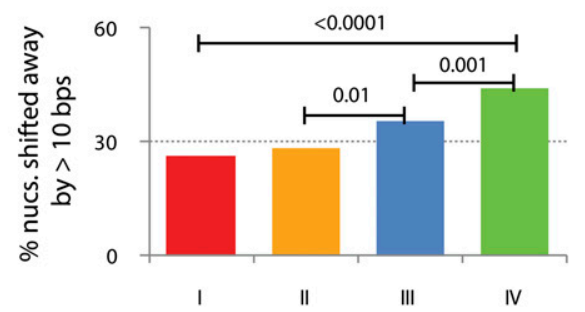

D
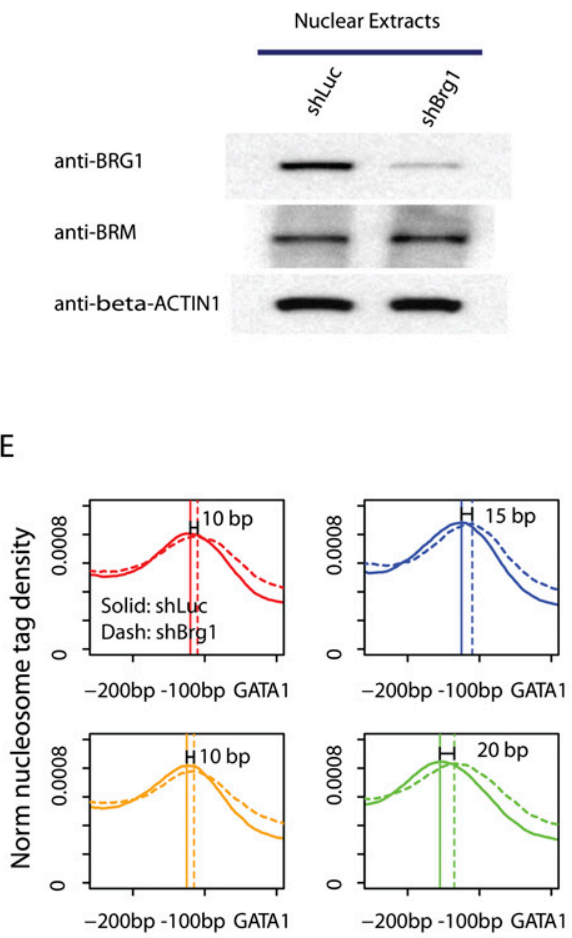

$\mathrm{F}$

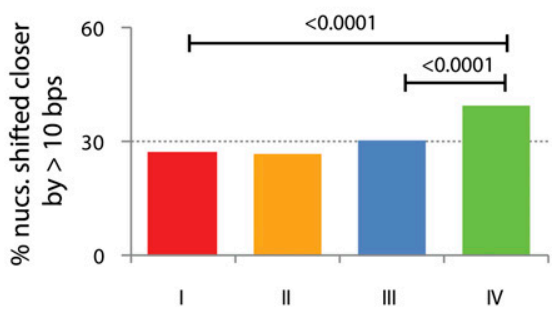

Figure 3. BRG1 mediates nucleosome shifting surrounding the GATA1 sites. (A) Nucleosome profiles in HSCs surrounding the distal GATA1 binding sites that are identified in $\mathrm{CD} 36^{+}$cells. The sites are grouped into four quartiles according to the level of BRG1 binding in CD $36^{+}$cells (I to IV denote low to high; 5951 sites per group). The profiles are organized such that the nearest nucleosome is in the upstream of the GATA1 site. Only sites that are predicted at linker regions in both cells are included. (B) Nucleosome profiles in CD36 $6^{+}$cells surrounding the same sites as in $A$. (C) The percentage of GATA1 binding sites exhibiting a nucleosome shift of $>10$ bp away from the sites during differentiation of HSCs. The GATA1 sites, which are located at linker regions and have nucleosomes within \pm 200 bp in both the HSCs and $C D 36^{+}$cells, are sorted into equal-sized groups ( $\sim 600$ per group) based on the BRG1 binding levels in the CD36 cells; I to IV denote the lowest to the highest. P-values are calculated by $\chi^{2}$ test. (D) BRG1 was efficiently knocked down, as shown by the Western blotting analysis of nuclear extracts from the CD36 $6^{+}$cell expressing a small hairpin RNA targeting BRG1 (shBrg1) or the luciferase gene (shLuc) with antibodies indicated on the left. ( $E$ ) Nucleosome profiles in BRG1 knockdown (dashed lines) and control cells (solid lines) for the region upstream of GATA1 binding sites, which are grouped as in $A$. ( F) The percentage of GATA1 binding sites exhibiting a nucleosome shift of $>10 \mathrm{bp}$ toward the sites after knocking down of BRG1 in the CD36 cells. The GATA1 sites, which are located at linker regions and have nucleosomes within $\pm 200 \mathrm{bp}$ in both the HSCs and CD $36^{+}$ cells, are sorted into equal-sized groups ( $\sim 700$ per group) based on the BRG1 binding levels in the CD $36^{+}$cells; I to IV denote the lowest to the highest.

sites that exhibited a nucleosome shift of $>10$ bp was significantly higher in the group with the highest BRG1 binding than the other groups with lower BRG1 binding (Fig. 3F). We then asked what fraction of the nucleosomes that were shifted away from the GATA1 sites during differentiation of HSCs exhibited a reverse shift after knocking down of BRG1 in $\mathrm{CD}^{2} 6^{+}$cells. Our data indicated that the quartile with the highest BRG1 binding showed the highest percentage $(57 \%)$ of the reverse nucleosome shift (Supplemental Fig. S4). In fact, all the other groups, regardless of the level of BRG1 binding, exhibited a higher percentage of reverse nucleosome shifting than what was expected by chance $(23 \% ; P$-value $<0.0001)$
(Supplemental Fig. S4). Together, these results indicated that BRG1 mediates nucleosome shifting at GATA1-bound enhancers to modulate the accessibility of the transcription factor binding sites during differentiation of HSCs to erythrocytes.

\section{BRGl-mediated nucleosome repositioning facilitates binding of TAL1 and subsequent transcriptional activation}

The BRG1-induced nucleosome shift during differentiation results in a longer nucleosome linker region surrounding the GATA1 sites, which may be important for GATA1 binding to establish functional 
enhancers. To test this hypothesis, we compared the genome-wide GATA1 binding in the BRG1 knockdown and control cells. Surprisingly, we did not see a significant decrease in GATA1 binding on a global level (Fig. 4A,B). Analysis of all GATA1 binding sites indicated that there was a similar number of sites with decreased or increased binding upon knocking down of BRG1 in CD36 ${ }^{+}$cells (Fig. 4C). These results indicated that although GATA1 binding is positively correlated with BRG1 binding, it does not require BRG1 for binding to its target sites.

Since TAL1, another key transcription factor for hematopoiesis (Porcher et al. 1996), may function with GATA1 as a complex (Osada et al. 1995; Wadman et al. 1997; Tripic et al. 2009; Kassouf et al. 2010), we mapped the genome-wide binding sites of TAL1 in CD36 ${ }^{+}$cells and found that the majority of GATA1 and TAL1 sites were colocalized in the genome (Fig. 4D). Interestingly, knocking down of BRG1 substantially decreased binding of TAL1 (Fig. 4E,F). In fact, $45.3 \%$ of all TAL1 sites showed significantly decreased binding of TAL1 upon knocking down of BRG1, while only $0.3 \%$ of the sites exhibited increased binding of TAL1 (Fig. 4G).

The above results are in favor of a model in which GATA1 binds to DNA and recruits BRG1 to facilitate subsequent TAL1 binding. However, TAL1 is known to bind to specific sites in mouse HSCs which lack GATA1 (Wilson et al. 2009, 2010). To understand this discrepancy, we mapped the genome-wide binding sites of TAL1 in human HSCs using ChIP-seq. Our data confirmed the binding of TAL1 to 12 out of 14 functional TAL1 sites validated using transgenic mice in a recent study (Wilson et al. 2009) (data not shown). However, the number of identified TAL1 binding sites in HSCs was only $16 \%$ of that in the CD36 ${ }^{+}$cells (Fig. 5A). In addition, two thirds of the sites in HSCs lost TAL1 binding during differentiation to erythrocytes. Motif analysis on the HSC-specific
TAL1 binding sites revealed "AGGAA" and "TGTGG" as the top motifs, which are recognized by SPI1/FLI1/ERG and RUNX1, respectively (Suppemental Fig. S5). These factors have been proposed to form a complex with TAL1 in mouse HSCs (Wilson et al. 2010). Remarkably, all these transcription factors were down-regulated when the cells differentiated into the $\mathrm{CD} 6^{+}$cells (Supplemental Fig. S5), supporting the notion that these HSC-specific TFs are involved in regulating the targeting of TAL1 in HSCs.

To investigate the function of BRG1 in TAL1 binding, we compared the TAL1 binding levels at three groups of TAL1 sites [HSC-specific (Group I); CD36 ${ }^{+}$-specific (Group II); constitutive in HSCs and CD36 ${ }^{+}$cells (Group III)]. We observed that the levels of TAL1 binding in the $\mathrm{CD} 36^{+}$cells were increased at the constitutive sites (Fig. 5B, green box vs. blue box in Group III), of which the majority (84\%) was accompanied with GATA1 binding. Compared to the $\mathrm{CD} 36^{+}$-specific sites, the constitutive sites were already associated with high levels of the BRG1 binding in HSCs (Fig. 5C, green box in Group II vs. green box in Group III), suggesting an open chromatin structure at the constitutive sites prior to differentiation. Although the BRG1 binding decreased modestly at the constitutive sites after differentiation (Fig. 5C, Group III), it was still higher than at the sites that lost TAL1 binding (Fig. 5C, blue boxes in Groups I and III), suggesting that an appropriate level of BRG1 binding is important for maintaining an open chromatin structure for TAL1 binding. Consistent with this idea, the TAL1 binding levels were significantly decreased by BRG1 knockdown at the constitutive sites, as at the $\mathrm{CD}^{+} 6^{+}$-specific sites (Fig. 5B, blue box vs. red box in Groups II and III).

To directly test the idea that the BRG1-mediated nucleosome shifting facilitates TAL1 binding, we extracted two groups of CD36 ${ }^{+}$-specific TAL1 binding sites that occurred at linker regions
A

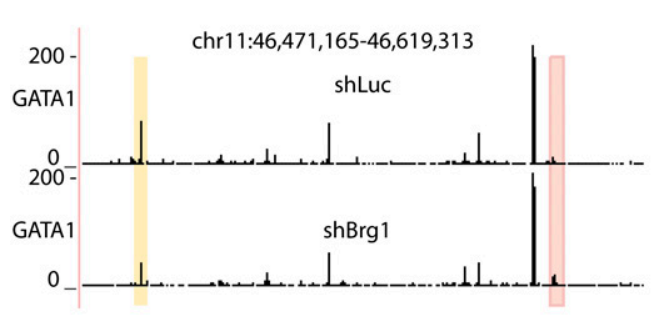

D

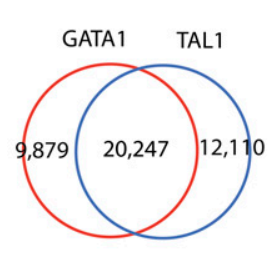

$\mathrm{E}$

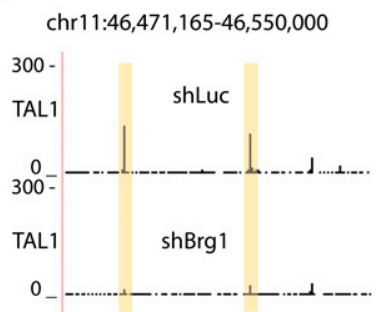

$\mathrm{B}$

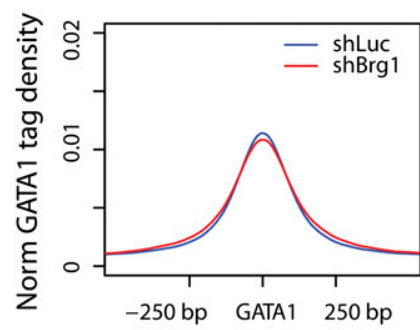

$\mathrm{F}$

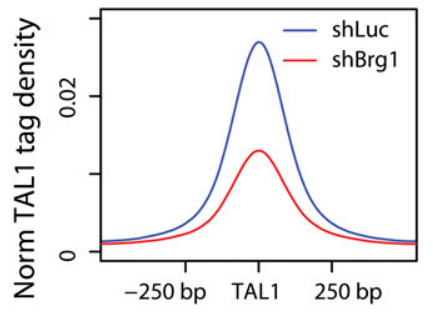

C

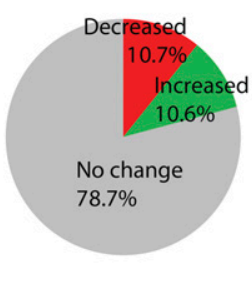

G

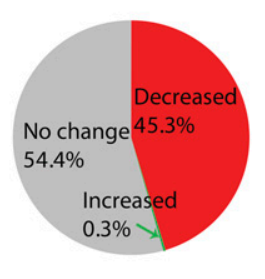

Figure 4. BRG1 knockdown decreases binding of TAL1 but not GATA1. (A) UCSC Genome Browser images of GATA1 binding in the BRG1 knockdown and control cells. Genomic regions with significantly increased and decreased levels of GATA1 binding $(P$-value $<0.00001 ;$ FC $>1.5)$ are highlighted in pink and yellow, respectively. (B) GATA1 tag densities around the GATA1 sites in the BRG1 knockdown and control cells. The tag density profiles of 39,702 GATA1-enriched regions are plotted for the control and knockdown cells. (C) The percentage of GATA1 binding sites that showed an increase, decrease, or no change in GATA1 binding in the BRG1 knockdown cells. (D) GATA1 and TAL1 are extensively colocalized in CD36 ${ }^{+}$cells. ( $E$ ) UCSC Genome Browser images showing that TAL1 binding was compromised in the BRG1 knockdown cells. Genomic regions with a significant decrease in TAL1 binding ( $P$-value $<0.00001 ; F C>1.5)$ are highlighted in yellow. $(F)$ TAL1 tag densities at the TAL1 sites in the BRG1 knockdown and control cells. The tag density profiles of 34,620 TAL1-enriched regions are plotted for the control and knockdown cells. (G) The percentage of TAL1 binding sites that showed an increase, decrease, or no change in TAL1 binding in the BRG1 knockdown cells.

1654 Genome Research

www.genome.org 
A

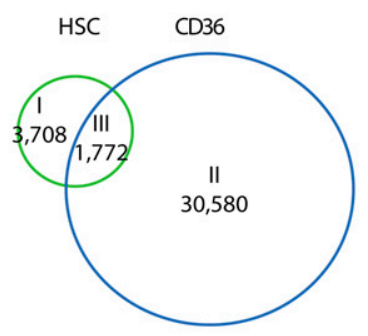

$\mathrm{D}$

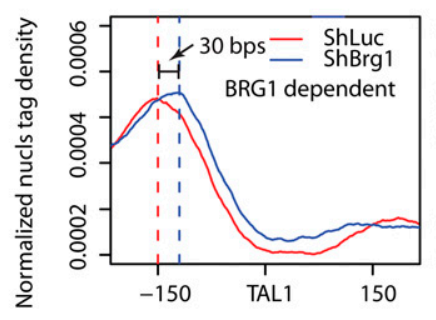

B

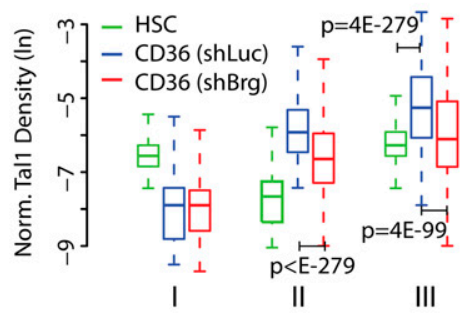

C

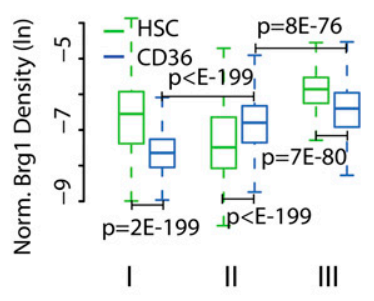

$\mathrm{F}$
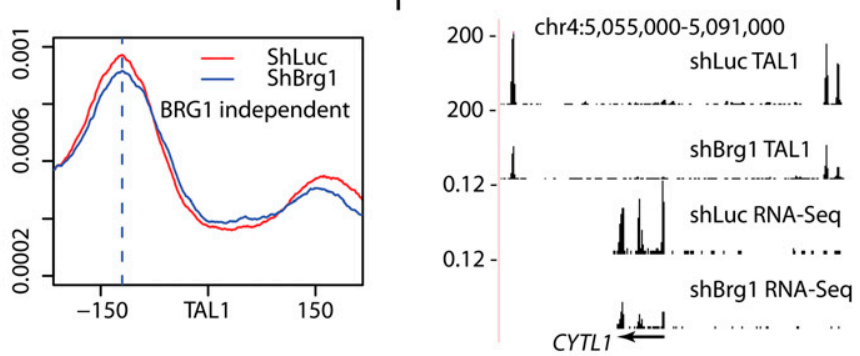

$\mathrm{E}$
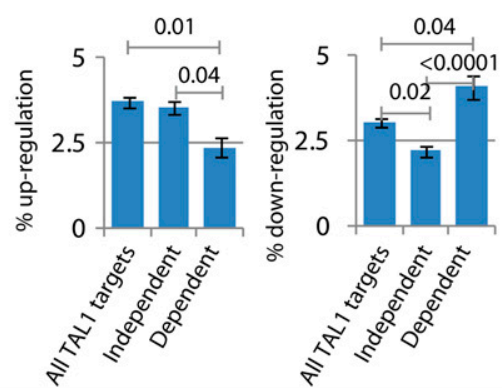

G

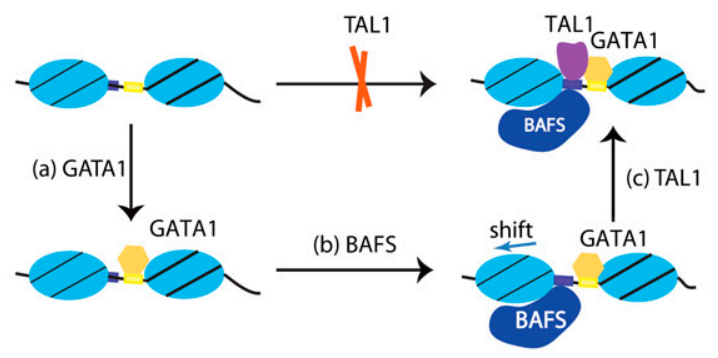

Figure 5. BRG1-induced nucleosome shifting facilitates binding of TAL1. (A) Venn diagram comparison of TAL1 binding sites in HSCs and CD36 ${ }^{+}$cells. (B) Box-plots for the normalized TAL1 tag density for the three groups of TAL1 sites as specified in $A$ in three cell types (HSCs, BRG1 knockdown CD36 ${ }^{+}$, and control CD36 ${ }^{+}$cells). (C) Box-plots for the normalized BRG1 density in HSCs and the CD $36^{+}$cells for the three groups of TAL1 binding sites as specified in $A$. $(D)$ Nucleosome profiles in BRG1 knockdown and control cells for the regions centered on the 6046 BRG1-dependent (left panel) or 1665 BRG1-independent TAL1 sites (right panel). See main text for the definition of "BRG1-independent" and "BRG1-dependent" TAL1 binding sites. ( $E$ ) Percentages of up-regulation and down-regulation among the genes that contain TAL1 binding sites in the region within $\pm 20 \mathrm{~kb}$ of the TSSs. Three groups of genes are shown: All TAL1 target genes (6905); Dependent (1542) indicates the group of genes to which TAL1 binding at all associated TAL1 sites was compromised by BRG1 knockdown; and Independent (3302) indicates the groups of genes to which TAL1 binding at none of the associated TAL1 sites was affected by BRG1 knockdown. The standard deviations are calculated by bootstrapping. $P$-values are calculated by $\chi^{2}$ test. ( $F$ ) UCSC Genome Browser images showing that the decreased binding of TAL1 is associated with decreased transcription of the CYTL1 gene measured by RNA-seq. The $y$-axis of RNA-seq tracks is normalized as number of tags per base pair sequence per million tags. (G) A model for the sequential activity of GATA1 binding, recruitment of BRG1, and nucleosome shifting, and binding of TAL1 to the hematopoietic enhancer regions.

in both HSCs and $\mathrm{CD}^{+} 6^{+}$cells. The first group was BRG1independent, which did not show a significant decrease in TAL1 binding after knocking down of BRG1 ( $P$-value $>0.01$; FC $<1.5$ ). The second group was BRG1-dependent, which showed a significant decrease in TAL1 binding after BRG1 knockdown $(P$-value $<$ 0.00001 ; FC > 1.5). Our analysis of nucleosome profiles in these two groups of TAL1 sites revealed that the flanking nucleosome shifted $\sim 30$ bp toward the TAL1 sites in the BRG1-dependent group, whereas no apparent nucleosome shift was detected in the BRG1-independent group (Fig. 5D). Similar results were obtained for the constitutive binding sites (Supplemental Fig. S6). Importantly, we observed no significant nucleosome shift even at the subset of the BRG1-independent TAL1 sites that were enriched with BRG1 binding (Supplemental Fig. S7). These results suggest that the nucleosome shifting is not likely just a byproduct of BRG1 binding, and the BRG1-mediated nucleosome shifting facilitates efficient binding of TAL1 to enhancers.

To test whether the decrease in TAL1 binding caused by inhibition of BRG1 leads to expression changes of TAL1 target genes, we examined the mRNA profiles in the BRG1 knockdown and control cells using RNA-seq. We were particularly interested in two subsets of putative TAL1 target genes, which were defined by the presence of TAL1 binding within $40 \mathrm{~kb}$ centered on transcription start sites (TSSs). The first subset (Dependent) consisted of genes to which TAL1 binding was significantly decreased by BRG1 knockdown, while the control subset (Independent) consisted of genes to which TAL1 binding was not significantly affected. We then calculated the percentages of up-regulated and down-regulated genes respectively for each group due to the BRG1 knockdown. Our data indicated that significantly more genes were down-regulated 
in the Dependent subset than in the Independent subset by knocking down of BRG1 (Fig. 5E, right panel; Fig. 5F), suggesting that BRG1-mediated TAL1 binding is mainly involved in gene activation. Although less frequent, we also observed that a minor fraction of up-regulated genes was correlated with loss of TAL1 binding by the BRG1 knockdown (Fig. 5E, left panel), suggesting a repressive role of the TAL1 binding in regulating their expression. However, BRG1 may also facilitate binding of other transcription factors to the TAL1-bound regions, and thus we cannot exclude the possibility that other factors may also contribute to the observed expression changes of TAL1 target genes in the BRG1 knockdown cells.

\section{Discussion}

In this study, we found that the nucleosomes flanking GATA1 sites located in the linker regions in HSCs were shifted away from the GATA1 binding sites during differentiation of HSCs to erythrocytes. We showed that BRG1 was associated with the majority of the GATA1-bound potential enhancers and was involved in the nucleosome shifting during differentiation of HSCs; knocking down of BRG1 resulted in a reverse shifting of the nucleosomes that shortened the nucleosome linker region, suggesting that constitutive activity of BRG1 is required to maintain the repositioned nucleosomes, which is similar to the activity of the yeast Iswi2 complex that repositions nucleosomes to block antisense transcription (Whitehouse et al. 2007).

It is interesting to speculate that BRG1 activity may induce nucleosome eviction or movement at the GATA1 sites overlapped with nucleosomes. However, the modest change of nucleosome profiles at these sites during differentiation (data not shown) does not allow us to draw a quantitative and solid conclusion due to the lack of reliable techniques and algorithms for a quantitative measurement of changes of nucleosome occupancy, since the results using MNase-seq depend on the degree of MNase digestion (Tirosh et al. 2010), and the chromatin sensitivity to MNase digestion varies between HSCs and the CD36 ${ }^{+}$cells (data not shown). Thus, to address this kind of question, further technical tools are required to quantitatively compare the nucleosome occupancy between different cell types or under different conditions.

BRG1 was recruited to proximal promoter regions, for example, in the interferon signaling pathways (Liu et al. 2002; Cui et al. 2004) and Toll-like receptor signaling pathways (Lai et al. 2009; Ramirez-Carrozzi et al. 2009). It was also detected at distal regulatory elements including those of the CIITA gene (Ni et al. 2008) and beta-globin gene (O'Neill et al. 1999; Im et al. 2005), where BRG1 may mediate gene activation by facilitating long-distance chromatin interaction (Kim et al. 2009b). These previous studies have proposed that BRG1 remodels nucleosome structure to increase the accessibility of chromatin and thus facilitates binding of a variety of transcription factors, for example, as shown for its requirement for binding of STAT3 to the IL6-inducible genes (Ni and Bremner 2007) and glucocorticoid receptor to a large number of its genomic sites (John et al. 2008). Although these studies appear to suggest a general requirement of BRG1 for binding of transcription factors to chromatin templates, it is unclear how ubiquitous BRG1 is selectively involved in lineage or cell type-specific transcription. Our data indicated that GATA1 binding to enhancers does not require BRG1. In contrast, the efficient binding of TAL1, which is colocalized with GATA1 in the genome, is dependent on the presence of BRG1, indicating that there is a remarkable selectivity of the transcription factors that functionally interact with BRG1. Although recent studies suggest that GATA1 collaborates exten- sively with TAL1 in erythroid differentiation (Kassouf et al. 2010), it is unknown how these two factors interplay with each other to execute the erythroid differentiation program. Our data revealed that GATA1-mediated recruitment of BRG1 and nucleosome repositioning regulate the binding of TAL1 to its target regulatory elements upon erythroid differentiation. These and previous data collectively suggest a sequential model that GATA1 serves as a pioneering factor by binding to its target sites in chromatin (Boyes et al. 1998; Kim et al. 2009b) [Fig. 5G, (a)], which recruits BRG1 to the enhancers via direct interaction (Kadam and Emerson 2003; Kim et al. 2009b) [Fig.5G, (b)]. Once associated with the target sites, BRG1 can reposition the flanking nucleosomes to generate a longer linker region, which allows TAL1 to bind to its target sites in the linker region and subsequently regulates transcription [Fig. 5G, (c)]. In summary, our data shed light on the interplay of the ATPdependent remodeling complexes, nucleosome organization, and tissue-specific transcription factors at enhancer regions, which is critical for transcriptional regulation during differentiation.

\section{Methods}

\section{Cell culture and ChIP-seq}

Primary human HSCs were isolated and differentiated to CD36 $6^{+}$ cells as described (Cui et al. 2009). The HSCs were infected with a lentiviral vector expressing GFP and shRNA targeting BRG1 or a control vector for two days. GFP-positive cells were sorted and further cultured for $8 \mathrm{~d}$ before being processed for ChIP-seq analysis using antibodies against BRG1 (Liu et al. 2001), H3K4me1 (abcam ab8898), GATA1 (abcam ab11852), TAL1 (Santa Cruz Biotechnology, sc-12984), and CTCF (Upstate, 07-729). ChIP and ChIP-seq assays were performed as described previously (Barski et al. 2007). Nucleosome positions in BRG1 knockdown and control cells were determined using MNase-seq (Schones et al. 2008). RNA-seq experiments for HSCs and $\mathrm{CD}^{2} 6^{+}$cells were carried out as described previously (Chepelev et al. 2009). The ChIP-seq data for $\mathrm{H} 3 \mathrm{~K} 4 \mathrm{me} 1$ for HSCs and $\mathrm{CD}^{+} 6^{+}$cells are from previous publication (Cui et al. 2009).

\section{Data analysis}

\section{ChIP-seq tag-enriched regions}

Sequence reads of $25 \mathrm{bp}$ were obtained from the ChIP-seq libraries on an Illumina Genome Analyzer, mapped to human genome (hg18) and processed as described previously (Barski et al. 2007). The ChIP-enriched peaks were identified by MACS for CTCF, GATA1, and TAL1 (Zhang et al. 2008a) and by SICER for BRG1 with default settings (Zang et al. 2009). To define genomic regions that showed a significant decrease or increase in TF binding after knocking down of BRG1, the MACS-predicted regions were pooled from the knockdown and control libraries. Then a submodule of the SICER program was employed to select regions that show significant difference in the tag counts between the two cells ( $P$-value $<0.00001$; FC $>1.5$ ).

\section{Tag density normalization}

The densities of short sequence tags of nucleosomes, CTCF, GATA1, TAL1, BRG1, and H3K4me1 were normalized to the library size and the number of sites, namely the number of tags per base pair per million reads. The $5^{\prime}$ tag and $3^{\prime}$ tags were shifted by $+75 \mathrm{bp}$ and $-75 \mathrm{bp}$, respectively, and merged to obtain the density. The average profiles in Figure 1A-C,E, Figure 3A,B,E, Figure 4B,F, Figure 5D, and Supplemental Figures S3, S6, and S7 were smoothed using 
a 50-bp sliding window. The nucleosome profiles at the UCSC Genome Browser in Figure 1D was smoothed using a Gaussian kernel with bandwidth of 15-bp.

To correct systematic bias in the nucleosome density between HSCs and CD $36^{+}$cells, we chose the nucleosome tag-enriched regions within $\pm 5 \mathrm{~kb}$ around constitutive CTCF sites as reference sets. A scale factor was calculated to make the mean densities be equal between the two sets and was applied to scale the tag density shown in Figure 1A-C,E and Supplemental Figure S3.

\section{Nucleosome tag-enriched regions}

Nucleosome tag-enriched regions were identified by the NPS Python software package (Zhang et al. 2008b). The program identified 1.7, 2.2, and 2.3 million nucleosome tag-enriched regions with a length varying from 80 to $250 \mathrm{bp}$ for HSCs $(P<0.05)$, and shLuc $\mathrm{CD}^{2} 6^{+}$and shBRG1 CD36 ${ }^{+}$cells $(P<0.01)$, respectively. To separate TF sites based on whether or not the sites are occupied by positioned nucleosomes, we determined the most likely nucleosome position in a nucleosome-enriched region $j$ as $p^{j}=\operatorname{sum}$ of $p^{j i{ }_{*}} n^{j i} /$ sum of $n^{j i}$, where $n^{j i}$ is the number of tags at position $p^{j i}$ in region $j$. The binding sites were being occupied by nucleosomes if the distance to the nearest nucleosome is $<75 \mathrm{bp}$.

\section{mRNA expression level}

To quantify the mRNA level of a gene, we followed the proposed RPKM measurement, that is, read per kilobase of exon model per million reads (Mortazavi et al. 2008). Differentially expressed genes were identified by EdgeR ( $P$-value $<0.001 ; \mathrm{FC}>2)$ (Robinson et al. 2010). For up-regulated (down-regulated) genes from status A to $\mathrm{B}$, those with RPKM less than 3 in $\mathrm{B}(\mathrm{A})$ are excluded, where $\mathrm{A}$, $\mathrm{B}=\left[\right.$ HSCs, CD36 $\left.{ }^{+}, \mathrm{BRG} 1 \mathrm{KD}\right]$ and $\mathrm{A} \neq \mathrm{B}$.

\section{Motif finding}

We used the MEME suite to define binding motifs at the GATA1 and TAL1 binding sites and determined their occurrences across the ChIP-seq tag-enriched regions (Bailey et al. 2006). Briefly, sequences of $100 \mathrm{bp}$ upstream of and $100 \mathrm{bp}$ downstream from the summits of the top 200 enriched regions were extracted and supplied to MEME for motif discovery. Motifs that showed the consensus of "GATAA" were selected as the GATA1 motif. Finally, we used the FIMO tool to scan the motif across the GATA1 ChIP-seq tag-enriched regions to get potential GATA1 occurrence sites ( $p$-value < 0.001) (Bailey et al. 2006).

\section{Data access}

The sequence data from this study have been submitted to the NCBI Gene Expression Omnibus (GEO) (http://www.ncbi.nlm. nih.gov/geo/) under accession number GSE26501.

\section{Acknowledgments}

This work was supported by the Division of Intramural Research Program of the National Heart, Lung and Blood Institute, NIH (K.Z.), R01HL090589 and R01HL091929 (S.H.), and K22HL101950 (D.E.S.).

\section{References}

Armstrong JA, Bieker JJ, Emerson BM. 1998. A SWI/SNF-related chromatin remodeling complex, E-RC1, is required for tissue-specific transcriptional regulation by EKLF in vitro. Cell 95: 93-104.

Bailey, TL, Williams, N, Misleh, C, Li, WW. 2006. MEME: Discovering and analyzing DNA and protein sequence motifs. Nucleic Acids Res 34: W369-W373.
Barski A, Cuddapah S, Cui K, Roh TY, Schones DE, Wang Z, Wei G, Chepelev I, Zhao K. 2007. High-resolution profiling of histone methylations in the human genome. Cell 129: 823-837.

Boyes J, Omichinski J, Clark D, Pikaart M, Felsenfeld G. 1998. Perturbation of nucleosome structure by the erythroid transcription factor GATA-1. J Mol Biol 279: 529-544.

Bultman S, Gebuhr T, Yee D, La Mantia C, Nicholson J, Gilliam A, Randazzo F, Metzger D, Chambon P, Crabtree G, et al. 2000. A Brg1 null mutation in the mouse reveals functional differences among mammalian SWI/ SNF complexes. Mol Cell 6: 1287-1295.

Cairns BR. 2007. Chromatin remodeling: Insights and intrigue from singlemolecule studies. Nat Struct Mol Biol 14: 989-996.

Cantor AB, Orkin SH. 2002. Transcriptional regulation of erythropoiesis: An affair involving multiple partners. Oncogene 21: 3368-3376.

Cheng Y, Wu W, Kumar SA, Yu D, Deng W, Tripic T, King DC, Chen KB, Zhang Y, Drautz D, et al. 2009. Erythroid GATA1 function revealed by genome-wide analysis of transcription factor occupancy, histone modifications, and mRNA expression. Genome Res 19: 2172-2184.

Chepelev I, Wei G, Tang Q, Zhao K. 2009. Detection of single nucleotide variations in expressed exons of the human genome using RNA-Seq. Nucleic Acids Res 37: e106. doi: 10.1093/nar/gkp507.

Chi TH, Wan M, Zhao K, Taniuchi I, Chen L, Littman DR, Crabtree GR. 2002. Reciprocal regulation of CD4/CD8 expression by SWI/SNF-like BAF complexes. Nature 418: 195-199.

Cui K, Tailor P, Liu H, Chen X, Ozato K, Zhao K. 2004. The chromatinremodeling BAF complex mediates cellular antiviral activities by promoter priming. Mol Cell Biol 24: 4476-4486.

Cui K, Zang C, Roh TY, Schones DE, Childs RW, Peng W, Zhao K. 2009. Chromatin signatures in multipotent human hematopoietic stem cells indicate the fate of bivalent genes during differentiation. Cell Stem Cell 4: 80-93.

Fan HY, Trotter KW, Archer TK, Kingston RE. 2005. Swapping function of two chromatin remodeling complexes. Mol Cell 17: 805-815.

Fujiwara T, O'Geen H, Keles S, Blahnik K, Linnemann AK, Kang YA, Choi K, Farnham PJ, Bresnick EH. 2009. Discovering hematopoietic mechanisms through genome-wide analysis of GATA factor chromatin occupancy. Mol Cell 36: 667-681.

Gutierrez J, Paredes R, Cruzat F, Hill DA, van Wijnen AJ, Lian JB, Stein GS, Stein JL, Imbalzano AN, Montecino M. 2007. Chromatin remodeling by SWI/SNF results in nucleosome mobilization to preferential positions in the rat osteocalcin gene promoter. J Biol Chem 282: 94459457.

He HH, Meyer CA, Shin H, Bailey ST, Wei G, Wang Q, Zhang Y, Xu K, Ni M, Lupien M, et al. 2010. Nucleosome dynamics define transcriptional enhancers. Nat Genet 42: 343-347.

Ho L, Jothi R, Ronan JL, Cui K, Zhao K, Crabtree GR. 2009. An embryonic stem cell chromatin remodeling complex, esBAF, is an essential component of the core pluripotency transcriptional network. Proc Natl Acad Sci 106: 5187-5191.

Im H, Grass JA, Johnson KD, Kim SI, Boyer ME, Imbalzano AN, Bieker JJ, Bresnick EH. 2005. Chromatin domain activation via GATA-1 utilization of a small subset of dispersed GATA motifs within a broad chromosomal region. Proc Natl Acad Sci 102: 17065-17070.

Imbalzano AN, Kwon H, Green MR, Kingston RE. 1994. Facilitated binding of TATA-binding protein to nucleosomal DNA [see comments]. Nature 370: $481-485$.

Jani A, Wan M, Zhang J, Cui K, Wu J, Preston-Hurlburt P, Khatri R, Zhao K, Chi T. 2008. A novel genetic strategy reveals unexpected roles of the SwiSnf-like chromatin-remodeling BAF complex in thymocyte development. J Exp Med 205: 2813-2825.

John S, Sabo PJ, Johnson TA, Sung MH, Biddie SC, Lightman SL, Voss TC, Davis SR, Meltzer PS, Stamatoyannopoulos JA, et al. 2008. Interaction of the glucocorticoid receptor with the chromatin landscape. Mol Cell 29: 611-624.

Kadam S, Emerson BM. 2003. Transcriptional specificity of human SWI/SNF BRG1 and BRM chromatin remodeling complexes. Mol Cell 11: 377389.

Kaplan N, Moore IK, Fondufe-Mittendorf Y, Gossett AJ, Tillo D, Field Y, LeProust EM, Hughes TR, Lieb JD, Widom J, et al. 2009. The DNAencoded nucleosome organization of a eukaryotic genome. Nature 458 : 362-366.

Kassouf MT, Hughes JR, Taylor S, McGowan SJ, Soneji S, Green AL, Vyas P, Porcher C. 2010. Genome-wide identification of TAL1's functional targets: Insights into its mechanisms of action in primary erythroid cells. Genome Res 20: 1064-1083.

Kim SI, Bresnick EH, Bultman SJ. 2009a. BRG1 directly regulates nucleosome structure and chromatin looping of the alpha globin locus to activate transcription. Nucleic Acids Res 37: 6019-6027.

Kim SI, Bultman SJ, Kiefer CM, Dean A, Bresnick EH. 2009b. BRG1 requirement for long-range interaction of a locus control region with a downstream promoter. Proc Natl Acad Sci 106: 2259-2264. 
Lai D, Wan M, Wu J, Preston-Hurlburt P, Kushwaha R, Grundstrom T, Imbalzano AN, Chi T. 2009. Induction of TLR4-target genes entails calcium/calmodulin-dependent regulation of chromatin remodeling. Proc Natl Acad Sci 106: 1169-1174.

Liu R, Liu H, Chen X, Kirby M, Brown PO, Zhao K. 2001. Regulation of CSF1 promoter by the SWI/SNF-like BAF complex. Cell 106: 309-318.

Liu H, Kang H, Liu R, Chen X, Zhao K. 2002. Maximal induction of a subset of interferon target genes requires the chromatin-remodeling activity of the BAF complex. Mol Cell Biol 22: 6471-6479.

Mortazavi A, Williams BA, McCue K, Schaeffer L, Wold B. 2008. Mapping and quantifying mammalian transcriptomes by RNA-Seq. Nat Methods 5: $621-628$.

Ni Z, Bremner R. 2007. Brahma-related gene 1-dependent STAT3 recruitment at IL-6-inducible genes. J Immunol 178: 345-351.

Ni Z, Abou El Hassan M, Xu Z, Yu T, Bremner R. 2008. The chromatinremodeling enzyme BRG1 coordinates CIITA induction through many interdependent distal enhancers. Nat Immunol 9: 785-793.

O’Neill D, Yang J, Erdjument-Bromage H, Bornschlegel K, Tempst P, Bank A. 1999. Tissue-specific and developmental stage-specific DNA binding by a mammalian SWI/SNF complex associated with human fetal-to-adult globin gene switching. Proc Natl Acad Sci 96: 349-354.

Osada H, Grutz G, Axelson H, Forster A, Rabbitts TH. 1995. Association of erythroid transcription factors: Complexes involving the LIM protein RBTN2 and the zinc-finger protein GATA1. Proc Natl Acad Sci 92: 95859589.

Porcher C, Swat W, Rockwell K, Fujiwara Y, Alt FW, Orkin SH. 1996. The T cell leukemia oncoprotein SCL/tal-1 is essential for development of all hematopoietic lineages. Cell 86: 47-57.

Ramirez-Carrozzi VR, Braas D, Bhatt DM, Cheng CS, Hong C, Doty KR, Black JC, Hoffmann A, Carey M, Smale ST. 2009. A unifying model for the selective regulation of inducible transcription by CpG islands and nucleosome remodeling. Cell 138: 114-128.

Robinson MD, McCarthy DJ, Smyth GK. 2010. edgeR: A bioconductor package for differential expression analysis of digital gene expression data. Bioinformatics 26: 139-140.

Schones DE, Cui K, Cuddapah S, Roh TY, Barski A, Wang Z, Wei G, Zhao K. 2008. Dynamic regulation of nucleosome positioning in the human genome. Cell 132: 887-898.

Tillo D, Kaplan N, Moore IK, Fondufe-Mittendorf Y, Gossett AJ, Field Y, Lieb JD, Widom J, Segal E, Hughes TR. 2010. High nucleosome occupancy is encoded at human regulatory sequences. PLOS ONE 5: e9129. doi: 10.1371/journal.pone.0009129.

Tirosh I, Sigal N, Barkai N. 2010. Widespread remodeling of mid-coding sequence nucleosomes by Isw1. Genome Biol 11: R49. doi: 10.1186/gb2010-11-5-r49.

Tripic T, Deng W, Cheng Y, Zhang Y, Vakoc CR, Gregory GD, Hardison RC, Blobel GA. 2009. SCL and associated proteins distinguish active from repressive GATA transcription factor complexes. Blood 113: 2191-2201.
Wadman IA, Osada H, Grutz GG, Agulnick AD, Westphal H, Forster A, Rabbitts TH. 1997. The LIM-only protein Lmo2 is a bridging molecule assembling an erythroid, DNA-binding complex which includes the TAL1, E47, GATA-1, and Ldb1/NLI proteins. EMBO J 16: 3145-3157.

Wang W, Cote J, Xue Y, Zhou S, Khavari PA, Biggar SR, Muchardt C, Kalpana GV, Goff SP, Yaniv M, et al. 1996. Purification and biochemical heterogeneity of the mammalian SWI-SNF complex. EMBO J 15: 53705382 .

Whitehouse I, Rando OJ, Delrow J, Tsukiyama T. 2007. Chromatin remodelling at promoters suppresses antisense transcription. Nature 450: $1031-1035$.

Wilson NK, Miranda-Saavedra D, Kinston S, Bonadies N, Foster SD, CaleroNieto F, Dawson MA, Donaldson IJ, Dumon S, Frampton J, et al. 2009. The transcriptional program controlled by the stem cell leukemia gene $\mathrm{Scl} / \mathrm{Tal1}$ during early embryonic hematopoietic development. Blood 113: $5456-5465$.

Wilson NK, Foster SD, Wang X, Knezevic K, Schutte J, Kaimakis P, Chilarska PM, Kinston S, Ouwehand WH, Dzierzak E, et al. 2010. Combinatorial transcriptional control in blood stem/progenitor cells: Genome-wide analysis of ten major transcriptional regulators. Cell Stem Cell 7: 532-544.

Wu JI, Lessard J, Olave IA, Qiu Z, Ghosh A, Graef IA, Crabtree GR. 2007. Regulation of dendritic development by neuron-specific chromatin remodeling complexes. Neuron 56: $94-108$.

Wurster AL, Pazin MJ. 2008. BRG1-mediated chromatin remodeling regulates differentiation and gene expression of T helper cells. Mol Cell Biol 28: 7274-7285.

Yu M, Riva L, Xie HF, Schindler Y, Moran TB, Cheng Y, Yu DN, Hardison R, Weiss MJ, Orkin SH, et al. 2009. Insights into GATA-1-mediated gene activation versus repression via genome-wide chromatin occupancy analysis. Mol Cell 36: 682-695.

Zang C, Schones DE, Zeng C, Cui K, Zhao K, Peng W. 2009. A clustering approach for identification of enriched domains from histone modification ChIP-Seq data. Bioinformatics 25: 1952-1958.

Zhang Y, Liu T, Meyer CA, Eeckhoute J, Johnson DS, Bernstein BE, Nussbaum C, Myers RM, Brown M, Li W, et al. 2008a. Model-based analysis of ChIP-Seq (MACS). Genome Biol 9: R137. doi: 10.1186/gb2008-9-9-r137.

Zhang Y, Shin H, Song JS, Lei Y, Liu XS. 2008b. Identifying positioned nucleosomes with epigenetic marks in human from ChIP-Seq. BMC Genomics 9: 537. doi: 10.1186/1471-2164-9-537.

Zhao K, Wang W, Rando OJ, Xue Y, Swiderek K, Kuo A, Crabtree GR. 1998. Rapid and phosphoinositol-dependent binding of the SWI/SNF-like BAF complex to chromatin after T lymphocyte receptor signaling. Cell 95: 625-636.

Received January 20, 2011; accepted in revised form June 21, 2011.

\section{Genome Research




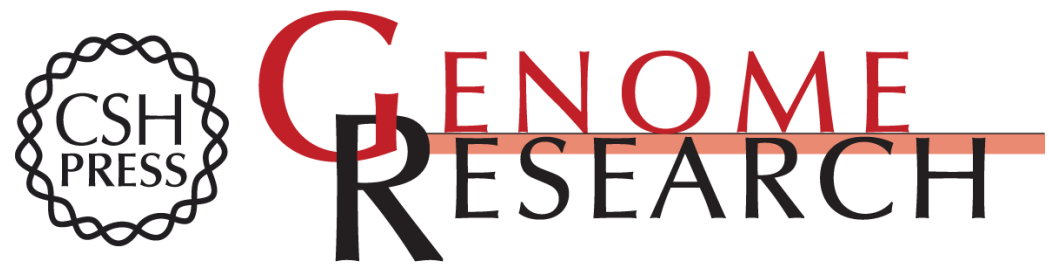

\section{Regulation of nucleosome landscape and transcription factor targeting at tissue-specific enhancers by BRG1}

Gangqing Hu, Dustin E. Schones, Kairong Cui, et al.

Genome Res. 2011 21: 1650-1658 originally published online July 27, 2011

Access the most recent version at doi:10.1101/gr.121145.111

Supplemental Material

\section{Related Content}

References

This article cites 53 articles, 17 of which can be accessed free at:

\section{License}

Email Alerting Service http://genome.cshlp.org/content/21/10/1650.full.html\#ref-list-1

Articles cited in:

http://genome.cshlp.org/content/21/10/1650.full.html\#related-urls

Dynamics of the epigenetic landscape during erythroid differentiation after GATA1 restoration

Weisheng Wu, Yong Cheng, Cheryl A. Keller, et al.

Genome Res. October , 2011 21: 1659-1671

Receive free email alerts when new articles cite this article - sign up in the box at the top right corner of the article or click here.

\section{Affordable, Accurate} Sequencing.

To subscribe to Genome Research go to: https://genome.cshlp.org/subscriptions 